\title{
Fracturas vertebrales infecciosas. A propósito de dos
} casos.

\section{cc) (1)(2)(2) \\ Infectious vertebral fractures. About two cases.}

Juan Carlos Salazar Flores. ${ }^{1}$, Edwin Javier Correa Vivanco. ${ }^{2}$, Katherine Elizabeth Gonzaga Aguilar. ${ }^{3}$, Gala Dennis Vasquez Hojas. ${ }^{4}$

\begin{abstract}
.
DOI: https://doi.org/10.33262/cienciadigital.v3i4.1.981

Tuberculosis is extrapulmonary, it occurs in 10 to $20 \%$ of non-immunosuppressed patients, and although the involvement is more frequent, it is in the lung, but it can affect any organ and cause severe damage to the infected one. Vertebral location is the most common. The problem is that in our country despite having adequate medication to treat it and the free treatment, most patients leave the antifungal treatment due to lack of awareness. The methodology of this research was compilation of information from a clinical history in a private clinic, follow its evolution in hospital and extra hospital, in addition to subsequent control of its complications. The main objective of this study is to help our doctors to raise awareness among patients that it is not only important to know how to treat tuberculosis but to finish it in its entirety to avoid drug resistance. Below are two cases of a patient of different ages from the Ecuadorian coast, with extrapulmonary tuberculosis who left the treatment and presented complications. We hope to conclude and bring the patient awareness to finish the antifungal treatment and the early detection of signs and symptoms of complications from leaving the medication.
\end{abstract}

Keywords: Infectious vertebral fracture, Pott's disease, treatment.

\section{Resumen}

La tuberculosis es extrapulmonar ocurre entre el 10 a $20 \%$ de los pacientes no inmunodeprimidos, y aunque la afectación es más frecuente es en el pulmon, pero

\footnotetext{
${ }^{1}$ Clinica Guayaquil, Guayaquil Ecuador, juank8sf@ hotmail.com

2 Clinica Guayaquil, Guayaquil Ecuador, edcorreavivanco85@hotmail.com

${ }_{3}^{3}$ Clinica Guayaquil, Guayaquil Ecuador, kelizaga@hotmail.com

${ }^{4}$ Clinica Guayaquil, Guayaquil Ecuador, Galinova_@hotmail.com
} 
puede afectar cualquier órgano y provocar daño severo al mismo que infecta. la localización vertebral es la más común. El problema es que en nuestro país a pesar contar con medicamento adecuado para tratarla y la gratuidad de su tratamiento, la mayoría de los paciente deja el tratamiento anti fímico por la falta de concientización. La metodología de esta investigación fue recopilación de información de una historia clínica en una clínica privada, seguir su evolución intrahospitalaria y extra hospitalaria, además de control posterior de sus complicaciones. El objetivo principal de este estudio es ayudar a nuestros médicos a incitar la concientización a los pacientes de que no solo es importante el saber tratar la tuberculosis sino terminarlo en su totalidad para evitar resistencias medicamentosas. A continuación se presenta dos casos de una paciente de diferentes edades de la costa ecuatoriana, con tuberculosis extrapulmonar el cual dejaron el tratamiento y presentaron complicaciones. Es importante la concientización al paciente para que finalice el tratamiento anti fímico y la detección temprana de signos y síntomas de complicaciones por dejar la medicación.

Palabras clave: Fractura vertebral infecciosa, mal de Pott, tratamiento.

\section{Introducción}

Las fracturas vertebrales por causas infecciosas es la forma mas comun de la tuberculosis extrapulmonar es la que afecta a la columna vertebral. Las vértebras lumbares superiores y torácicas inferiores son las regiones más afectadas desde T8 hasta L3 (Eisen, Honywood, Shingadia, \& Novelli, 2012); (Fuentes Ferrer, Gutiérrez Torres, Ayala Ramírez, Rumayor Zarzuelo, \& del Prado González, 2012) Para algunos autores las principales articulaciones que se afectan son: coxofemorales, rodillas, sacroilíacas, tobillos, muñecas y codos; mientras que para otros, las artritis se localizan de forma característica en las articulaciones sacroilíacas y esternoclaviculares, seguidas en orden de frecuencia de la sínfisis del pubis, la rodilla, la coxofemoral y otras (esternocostal, escapulohumeral y la muñeca). La afección poliarticular es rara. (Cepero Morales, Martínez Larrate, Sosa Almeida, \& Molinero Rodríguez, 1998).

También se le conoce como espondilitis tuberculosa, tiene su origen en un foco primario pulmonar (excepcionalmente urogenital), que favorece uno secundario a nivel óseo, en especial en la columna vertebral. El Mycobacterium tuberculosis alcanza la columna por vía hematógena o a través de los linfáticos prevertebrales. La lesión produce una destrucción vertebral progresiva que ocasiona al paciente un dolor continuo. Esta puede evolucionar desfavorablemente si no se diagnostica precozmente y ocasionar graves deformidades en la columna e importantes lesiones medulares. (Iseman, 1998); (Aguado García, 2002)

El medio de diagnostico por imagen es el pilar fundamental para el diagnostico y el tratamiento definitivo, por ejemplo la radiografia simple diagnostica $91-99 \%$, la tomografia 
diagnostica $47 \%$ de los casos dando con exactitud la destruccion osea fragmentaria, en 34 $\%$ osteolitica, esclerotica en $10 \%$ y en $30 \%$ subperiostica. La resonancia magnetica es el medio de diagnostico de eleccion ya que permite visualizar la totalidad de cambio en las vertebras y ademas de abcesos en tejidos adyacentes. (Zamora, Ramirez, \& Pantoja , 2014); (Gonzales, y otros, 2010).

En el Ecuador, el Diario El Telégrafo (2017) público que hasta el 2016 se reportaron 10,4 millones de personas enfermas de Tuberculosis, y en el 2015 murieron 1,8 millones con este mal en el Ecuador. La mayor concentración de casos está en la provincia del Guayas con el $48,25 \%$ de los casos nuevos de todas las formas de Tuberculosis. Es relevante observar que no existe estadística específica de Tuberculosis Vertebral en el país. Ecuador ocupa el sexto lugar en el mundo de vulnerabilidad, según el Programa de Estrategia Control de la Tuberculosis.

\section{Importancia del problema}

Describir que en nuestro pais a pesar de tener grandes altas de mortalidad de esta enfermedad no se ha preparado a personal de salud para identificacion oportuna de signos y sintomas de tuberculosis extrapulmonar. Ademas dar a entender que el personal de salud debe dar informacion importante a la comunidad para poder evitar compliaciones por resistencia a medicamentos antifimicos.

\section{Caso clinico numero 1}

Paciente femenina de 58 años de edad cuya procedencia es Quevedo, con lateralidad diestra, profesa, religion catolica. Antecedentes patologicos personales de hipertension arterial de reciente diagnostico, que refiere cuadro clínico de aproximadamente un mes y medio de evolución caracterizado por dolor lumbar de inicio súbito sin causa aparente con irradiación hacia miembros inferiores que dificulta la marcha, el mismo que aumenta 4 días despues por lo que decide a su ingreso a hospital IESS Quevedo donde se la cataloga como radiculopatía a través de exámenes de imagen y se la transfiere a esta unidad medica en Guayaquil para valoracion y tratamiento especializado, dentro de los examen fisico se encuentra paciente con tensiones arteriales altas ( $160 / 90)$, con parestesia e hipotonia con hiporreflexia de miembros inferiores bilateral, examenes de laboratorio con leucocitos 7500 , neutrofilos de $75 \%$, la tomografia 3D de columna vertebral el cual revelo discopatia degenerativa con neumodiscos en L4 - L5, alteracion de la morfologia de la densidad y cuerpos vertebrales D9 - D10, con lesiones osteoliticas en los platillos vertebrales correspondientes, tal como se observa en la figura 1. Resonancia Magnetica de columna dorsal, la secuencia T2 Demuestran incremento de intensidad de señal con alteracion de cuerpos vertebrales D9 - D10, con colección de partes blandas en la region paravertebral, ademas exite otra imagen laminar hiperintensa sub - ligamentosa en forma de colección laminar que comprime el cordon medular, presencia de señal de liquido en el espacin intervertebral de D9 - D10 con compromiso del Disco. En 
secuencia T1 contrastado con Gadolineo muestran realce irregular y heterogeneo en los planos blandos paravertebrales y platillos terminales D9 - D10, tal como se observa en figura 2. Debido a el dolor neurocirugia recomienda colocacion de opiode (tramal en infusion) y la colocacion de corset de Jewett, y pasa a quirofano para realizacion de biposia de tejido adjunto a lesiones medulares el cual revela la presencia de bacilo tuberculosos en colecciones paravertebrales adquirida dando asi el diagnostico de espondilitis tuberculosa. Se solicita valoracion de infectologo por lo que se inicia tratamiento antifimico, Linezolid, levofloxacino y amikacina. Paciente posteriormente no controla efinteres urinario por lo que se realizacion de citostomia. Evoluciona favorablemente posterior a cistostomia el cual se decide dar alta medica y que continue controles ademas de tratamiento antifimico a centro de salud del cual fue referido.

\section{Caso clinico numero 2}

Paciente masculino de 17 años de edad, procedencia de Santa Elena, lateralidad diestra, religion Catolica con antecedentes patologicos de adenopatias (dos) en region cervical no dolorosas hace 3 meses de evolucion con exceresis y envio de patologia de ganglio cervical el cual revela la presencia de bacilos tuberculosos en glanglio y que resive tratamiento antifimico. Refiere cuadro clínico de 3 meses de evolución caracterizado por debilidad generalizada que se acompaña de astenia, anorexia y dificultad para la marcha. El presente cuadro clínico se exacerba hace 14 días previas a su ingreso a nuestra unidad asistencial, valorado por Hospital Liborio Panchana Provincia de Santa Elena donde posterior a imágenes y exámenes realizados se determina tuberculosis de presunto origen vertebral y se lo transfiere a clinica particular para descompresión medular por parte de neurocirugía, examen fisico signos vitales sin alteraciones de rangos normales, en miembros inferiores se observa con parestesia, y paraplejia e hipotonia con hiporreflexia de miembros inferiores bilateral, examen de laboratorio revelo leucocitos 9600 co neutrofilos de $88 \%$, HIV no reactivo, se decide realizar tomografia reconstructiva 3D que revelo luxofractura de las vertebras D10 D11 con compresion de canal raquideo. Lesiones osteoliticas de varios cuerpos vertebralesdorsales y casi de todas las vertebras lumbales y de las primeras sacras, con colecciones paravertebrales, ademas se observa compromiso de hueso iliaco y acetabulo del lado derecho, tal como se observa en figura numero 3. Se solicia interconsulta con el depatamento de neurocirugia el cual realiza fijacion transpendicular con placa metalica a nivel de vertebras T7 a T 12 + laminectomia + colocacion de injerto oseo, tal como se observa en imagen 4. Ademas de toma de muestra de tejido vertebral el cual no $\mathrm{KOH}$ no se observa estructuras fungicas compatibles con levaduras ni presencia de bacilos tuberculosos, paciente que durante el post operatorio presenta fiebre por lo que se envia antipireticos. Se recomienda colocacion de corset de Jewett. Paciente posterior de 10 dias en cuidados intensivos presenta mejoria por lo que deciden enviarlo a unidad de cuidados intermedios, el cual no se observa mejoria de su paraplejia. Posterior de 21 dias pos quirurgico, sin 
realizacion de fiebre ni presencia de dolor se decide dar alta medica con referencia a hospital de origen para continiudad de tratamiento antifimico.

Imagen 1. Tomografia de Columna total con reconstruccion 3D. Revela Alteracion de morfologia de la densidad y lesiones osteoliticas en los platillos de los cuerpos vertebrales D9 y D10
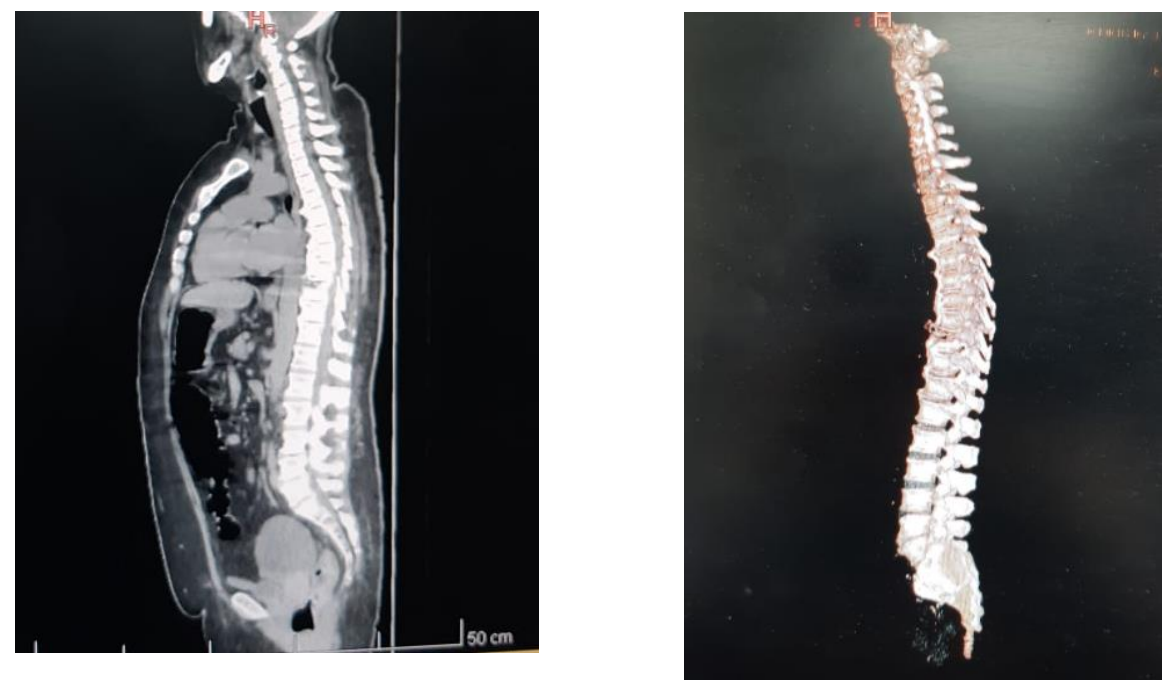

Fuente: Edwin Javier Correa Vivanco

Imagen 2. Resonancia Magnetica en secuencia T1 contrastada que muestra moderado realce irregular y heterogeneo en los planos blandos paravertebrales y en los palillos terminales D9 - D10

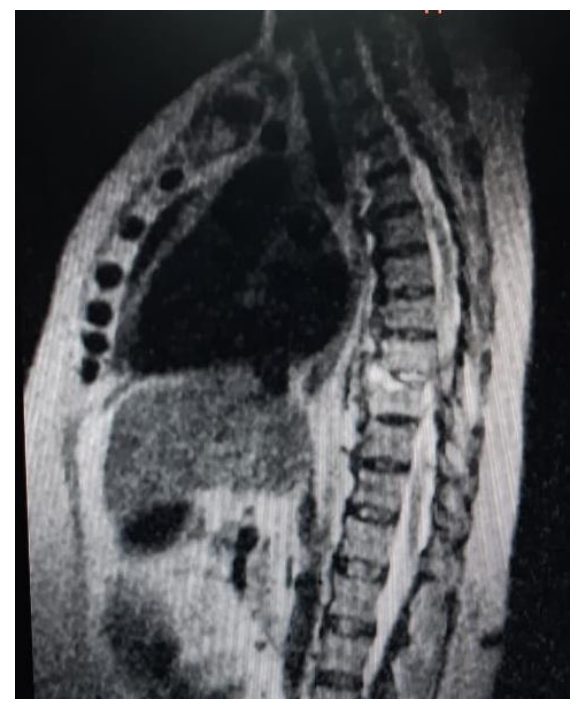

Fuente: Edwin Javier Correa Vivanco 
Imagen 3. Tomografia de columna dorsolumbar 3D se observa Luxofractura de las vertebras D10 - D11 con compresion de canal raquideo
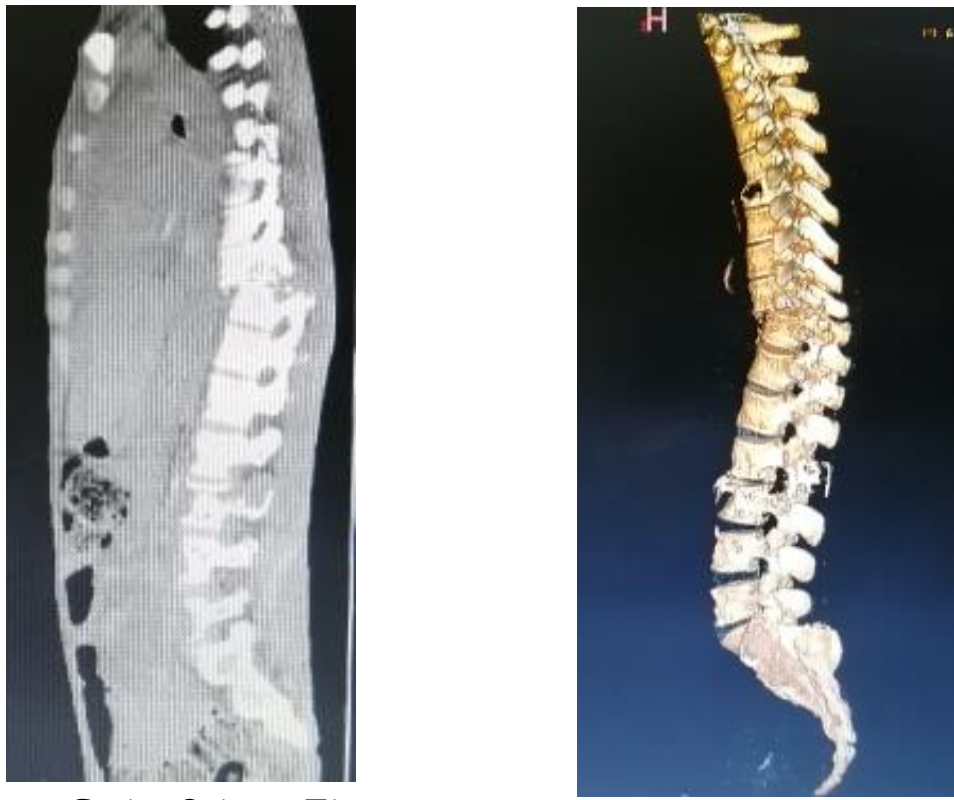

Fuente: Juan Carlos Salazar Flores

Imagen 4. Tomografia de columna Dorsolumbar posterior a fijacion transpendicular con placa metalica a nivel de vertebras T7 a T $12+$ laminectomia + colocacion de injerto os
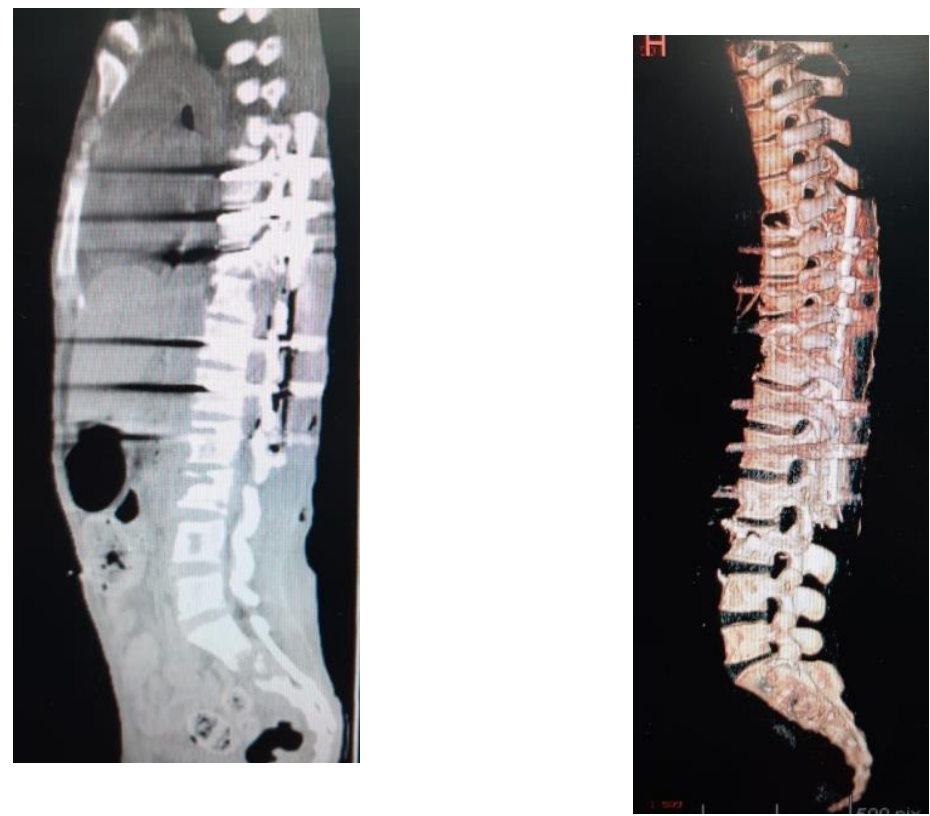

Fuente: Edwin Javier Correa Vivanco 


\section{Discusion}

Las afectaciones de columna lumbar son secundarias a infecciones primarias pulmonares, el dolor lumbar constituye el síntoma inicial notificado con mayor frecuencia, tal como ocurrió en el caso anteriormente expuesto. Existen informes donde este dolor se presentó en $86 \%$ de los casos, mientras que la fiebre solo se halló en 35-60\%, probablemente porque los pacientes, por lo general, toman analgésicos. (Fanlo \& Tiberio, 2007); (www. elsevier.es, 2006)

El problema del diagnóstico diferencial de esta afección es difícil, debido a que se presenta con cuadros dolorosos muy variados, por ello es tan frecuente que la espondilitis póttica transcurra inadvertida bajo los más extraños diagnósticos.

Cuando se confirma la existencia de esta afección primero se debe encaminar al paciente. Ningún sostén externo ni corsé pueden sustituir al reposo en cama, cualquiera que sea la gravedad y la localización del foco de espondilitis. (Marco Puche, Lopez Montesinos, Calvo Penades, Salvaret LLeti, \& Gonzales Puig, 2009)

El tratamiento es fundamentalmente médico con fármacos tuberculostáticos (isoniacida, rifampicina, pirazinamida o etambutol) habituales para la infección tuberculosa pulmonar con una duración media de 12 meses (Trecarichi, Di Meco, Mazzotta, \& Fantoni, 2012)

En cuanto a la evolución de esta enfermedad se plantea, que no suele tener tendencia progresiva solamente en los casos de profunda decadencia orgánica, pues trae consigo extensos aplastamientos vertebrales. Frecuentemente ocurre, que estas lesiones se mantienen estacionarias y se presentan fenómenos de reacción defensiva.

\section{Conclusiones}

- Es importante tener en cuenta la sospecha clínica sobre esta condición en cualquier edad con contacto positivo para tuberculosis, no sólo en inmunosuprimidos. De esta manera, se podrá realizar un diagnóstico temprano y un pronto inicio de la terapia antibiótica antituberculosa combinada. Se requiere más evidencia para evaluar la prevalencia de las diferentes manifestaciones clínicas y se necesitan guías de estandarización terapéutica para su manejo.

- Es importante de parte del personal medico la concientizacion a los pacientes de finalizar el esquema antifimico ya que es importante para evitar la resistencia y complicaciones extrapulmonares 


\section{Referencias Bibliograficas}

- Eisen S, Honywood L, Shingadia D, Novelli V. Spinal tuberculosis in children. Arch Dis Child. 2012;97(8):724-9. doi: 10.1136/archdischild-2011-301571.

- Fuentes Ferrer M, Gutiérrez Torres L, Ayala Ramírez O, Rumayor Zarzuelo M, del Prado González N. Tuberculosis of the spine. A systematic review of case series. Int Orthop. 2012;36(2):221-31. doi: 10.1007/s00264-011-1414-4.

- Cepero Morales RJ, Martínez Larrate JP, Sosa Almeida M, Molinero Rodríguez C. Osteoartritis tuberculosa. Rev Cubana Med 1998; 37(3):190-94. [consulta: 20 septiembre 2011].

- Iseman MD. Tuberculosis. Infecciones micobacterianas. En: Cecil. Textbook of Medicine. 21 ed. Philadelphia: WB Saunders, 1998:1724-32.

- Aguado García JM. Tuberculosis y otras infecciones por micobacterias. En: Medicina Interna. Enfermedades producidas por micobacterias. Barcelona: Masson, 2002:1789-97.

- Zamora T; Ramírez L; Pantoja A. (2014, Junio) Tuberculosis Vertebral y

- Compresión de la Médula Espinal. Bogotá. Vol. 36 No. 2 (105) Págs. 158-164

- González J; García J; Anibarro L; Vidal R; Esteban J; Blanquer R; Moreno S; Ruiz J. (2010). Documento de Consensos sobre diagnóstico, tratamiento y prevención de la tuberculosis. Vol.28 Num.5. Recuperado de: https://www.elsevier.es/es-revistaenfermedades-infecciosas-microbiologia-clinica-28-articulo-documento-consensosobre-diagnostico-tratamiento-S0213005X10001126

- Diario El Telégrafo-Ecuador (2017, Marzo). Recuperado de: http://www.eltelegrafo.com.ec/noticias/sociedad/4/hay-una-alta-incidencia-decasosde-tuberculosis-y-la-gente-se-automedica

- Fanlo P, Tiberio G. Tuberculosis extrapulmonar. An Sist Sanit Navar. 2007; 30(supl 2):143-62.

- 6. García-Cía JI, Esteban J. Infecciones osteoarticulares por microbacterias en un hospital universitario. Enferm Infecc Microbiol Clin. 2006 [citado 8 Dic 2011];24(10). Disponible en: http://www. elsevier.es/es-revista-enfermedadesinfecciosas-microbiologia-clinica $\quad$-28-articulo-infecciones-osteoarticulares-pormicobacterias-un-13095380

- 7. Marco Puche A, López Montesinos B, Calvo Penadés I, Salavert Lletí M, González Puig L. Osteomielitis tuberculosa espinal y artropatía de Poncet. An Pediatr (Barc). 2009; 71(4):365-6.

- E.M. Trecarichi, E. Di Meco, V. Mazzotta, M. Fantoni. Tuberculous spondylodiscitis: Epidemiology, clinical features, treatment, and outcome. Eur Rev Med Pharmacol Sci., 16 (2012), pp. 58-72 
PARA CITAR EL ARTÍCULO INDEXADO.

Salazar Flores, J., Correa Vivanco, E., Gonzaga Aguilar, K., \& Vasquez Hojas, G. (2019). Fracturas vertebrales infecciosas. A propósito de dos casos. Ciencia Digital, 3(4.1), 66-74. https://doi.org/10.33262/cienciadigital.v3i4.1.981

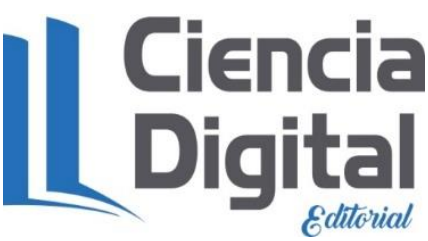

El artículo que se publica es de exclusiva responsabilidad de los autores y no necesariamente reflejan el pensamiento de la Revista Ciencia Digital.

El artículo queda en propiedad de la revista y, por tanto, su publicación parcial y/o total en otro medio tiene que ser autorizado por el director de la Revista Ciencia Digital.
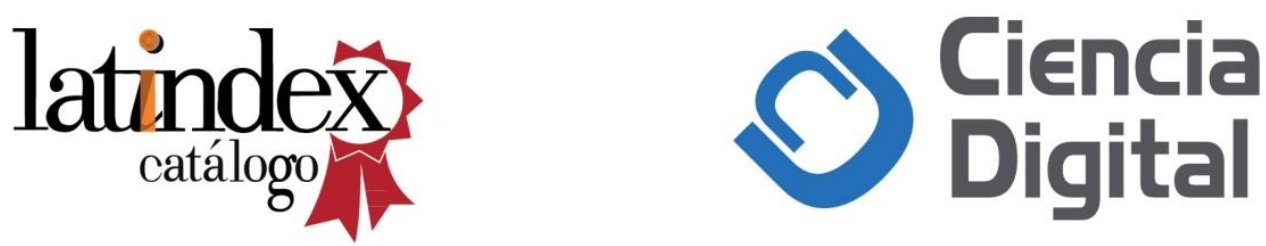